\section{Implants pour la délivrance de principes actifs}

Nicolas Blanchemain, Florence Siepmann, Juergen Siepmann

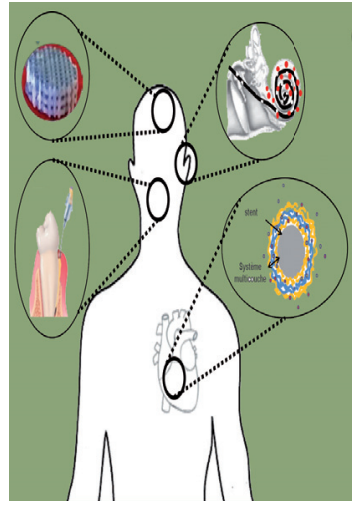

Université de Lille, Inserm, U1008, CHU de Lille, Controlled Drug Delivery Systems and Biomaterials, Faculté des Sciences Pharmaceutiques et Biologiques de Lille, F-59000 Lille, France. nicolas.blanchemain@ $\underline{\text { univ-lille2.fr }}$

II est à souligner que l'efficacité du principe actif dépend essentiellement de sa concentration au site d'action et ainsi du profil de sa libération. Une concentration de principe actif trop faible conduit à un échec du traitement (voire une aggravation: dans le cas des antibiotiques par exemple, cela peut conduire à l'émergence de souches bactériennes résistantes). Par contre, une concentration de principe actif trop importante provoque des effets indésirables. Ainsi, il est important de rester dans la «fenêtre thérapeutique du principe actif» (qui se situe entre la concentration minimale efficace et la contraction minimale toxique) (Figure 1). Si toute la dose de principe actif est libérée immédiatement, des effets toxiques peuvent apparaître (selon la dose administrée et la fenêtre thérapeutique) et la durée de l'effet thérapeutique peut être très courte (due à l'élimination du principe actif par le corps humain). En contrôlant la vitesse de libération du principe actif à partir de l'implant, des concentrations optimisées peuvent être atteintes au site d'action pendant de longues durées (Figure 1).

Cette revue présente quelques exemples de stratégies innovantes pour développer de nouveaux implants à libération contrôlée de principe actif dans différentes aires thérapeutiques de la parodontologie à l'oto-rhino-laryngologie (ORL) en passant par la chirurgie endo-vasculaire et la chirurgie maxillo-faciale.

\section{Les substituts osseux antibactériens}

L'hydroxyapatite (HA), constituant naturel principal de l'os, est largement utilisé comme substitut de reconstruction osseuse dans le cas du traitement de défects osseux $[2,46](\rightarrow)$.

Les disciplines chirurgicales intervenant sur l'os sont des chirurgies dites « à ciel ouvert» impliquant un risque d'infection. Celles-ci restent

$\rightarrow$ Voir la Synthèse de F. Jordana et al., page 60 de ce numéro 


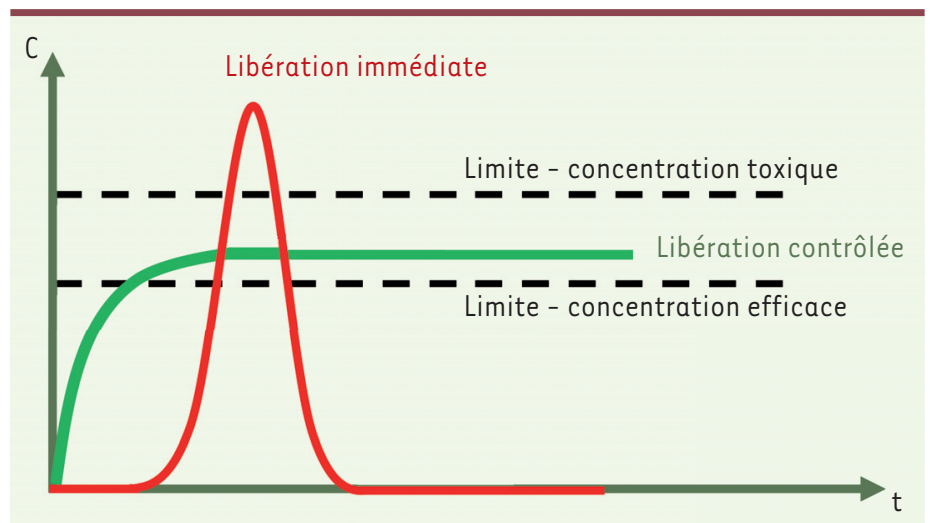

Figure 1. « Fenêtre thérapeutique 》 d'un principe actif et représentation schématique des profils de concentration d'un principe actif au site d'action suite à l'administration d'un système à libération immédiate (courbe rouge) et contrôlée (courbe verte). C : concentration du principe actif; $t$ : temps.

limitées ( 1 à $2 \%$ ) mais elles peuvent conduire à des conséquences dramatiques pour le patient (ré-interventions, expositions osseuses, amputation, fistulisation, etc.) [3, 4]. L'administration par voie systémique d'antibiotiques est la principale solution à ces infections mais la diffusion des principes actifs dans l'os étant plus faible que dans les autres tissus, les doses administrées doivent être élevées au risque d'atteindre, à un instant donné, une dose toxique [5]. De nombreuses voies de recherche ont été développées pour apporter des propriétés antibactériennes à l'hydroxyapatite (HA) en incorporant le principe actif dans la céramique en suspension, par compaction à froid ou encore par incorporation dans la porosité de l'HA [6]. La modification de surface par un polymère de cyclodextrine [7], par silanisation [8], ou à l'aide de bisphosphonate ou d'acides aminés [9], permettent l'adsorption de principes actifs mais avec un impact sur les propriétés chimiques et cristallines de la biocéramique. Une autre voie est la conception d'un composite céramique/polymère dans lequel le polymère comblerait la porosité de la céramique, libérerait un principe actif et se résorberait pour laisser place à l'os néosynthétisé [10, 11]. La structure du chitosan (CHT), issu de l'exosquelette des crustacés, est similaire à celle des glycosaminoglycanes (GAG) qui se trouvent dans la matrice extracellulaire (MEC) [12]. II présente également des propriétés hémostatiques, biocompatibles et bactériostatiques [13]. Grâce à sa fonction amine primaire, il a la capacité de former un complexe polyélectrolyte qui est un réseau réversible, formé par des liaisons non covalentes [14], ou des réseaux irréversibles, chimiquement formés par des liaisons covalentes. Pour former des liaisons covalentes et obtenir un hydrogel [15], I'utilisation d'agents réticulants, comme le glutaraldéhyde, le formaldéhyde, le tripolyphosphate, l'éthylène glycol, le diglycidyle éther, le diisocyanate ou la génipine, est nécessaire. La génipine est l'agent réticulant le moins cytotoxique, elle est donc la plus utilisée pour former des hydrogels chimiques [16]. La réaction de réticulation du chitosan (CHT) par la génipine (Gnp) implique deux mécanismes réactionnels qui aboutissent à l'apparition d'une coloration bleue [17]. La réaction de réticulation du chitosan est relativement lente, ce qui permet de pouvoir injecter la solution contenant le chitosan et la génipine dans une pièce en hydroxyapatite macroporeuse $e^{1}$ avant sa réticulation. La pièce est alors placée à $40{ }^{\circ} \mathrm{C}$ pendant 72 heures pour l'obtention d'un scaffold ${ }^{2}$ hybride chitosan/hydroxyapatite chargé de l'antibiotique, la ciprofloxacine ${ }^{3}$ (Figure 2A).

Après avoir formé le scaffold hydride, le profil de libération de la ciprofloxacine a été évalué en condition dynamique en flux continu ( $35 \mathrm{ml} / \mathrm{min}$ ) dans du tampon phosphate (à $\mathrm{pH} 7,4$ à $37^{\circ} \mathrm{C}$ ) (Figure 3 ). Les résultats montrent un profil de libération prolongée de l'antibiotique incorporé dans le scaffold hydride qui dépend de sa concentration initiale (Figure 2B). Ainsi, plus la concentration en ciprofloxacine dans la formulation est importante et plus le profil de libération sera prolongé passant de 20 à 80 minutes lorsque les concentrations varient de 0,1 à $1 \%$.

Ce scaffold hybride hydroxyapatite/chitosan à libération prolongée est prometteur pour le traitement des infections osseuses pour, à terme, permettre une meilleure repousse de l'os et sa vascularisation.

\section{Les stents 《 actifs 》}

L'athérosclérose, étiologie la plus fréquente des maladies cardio-vasculaires, peut être traitée par angioplastie avec la pose d'un stent ${ }^{4}$ métallique (bare metal stent ou BMS). Néanmoins, le traumatisme lié à la dilatation de l'artère et à la présence d'un corps étranger au sein de la paroi est à l'origine d'un processus inflammatoire local et de cicatrisation excessif pouvant mettre en danger le bon résultat de la revascularisation, initialement obtenue. Ainsi, une resténose intrastent (ou RIS) apparaît dans 20 à $30 \%$ des cas lorsque le stent est posé en position coronaire, et jusqu'à $50 \%$ s'il est en position périphérique. La première génération de stents actifs, basés sur la libération de molécules thérapeutiques (drug eluting stent, DES), a permis d'apporter une alternative intéressante aux résultats décevants obtenus avec les BMS en réduisant significativement le taux de resténose [18]. Néanmoins, ces stents conduisent à une augmentation importante des thromboses tardives aiguës [19] ayant pour origine l'augmentation du délai de cicatrisation de la paroi de l'artère et l'absence de réendothélialisation du segment artériel traité [20]. Par ailleurs, le recours à des molé-

\footnotetext{
${ }^{1}$ Collaboration avec le Dr Jean Christophe Hornez (Université de Valenciennes).

${ }^{2}$ Échafaudage, empilement.

${ }^{3}$ Un antibiotique appartenant à la famille des fluoroquinolones.

${ }^{4}$ Le stent (ou ressort) est un dispositif médical métallique utilisé pour maintenir ouvert une cavité.
} 


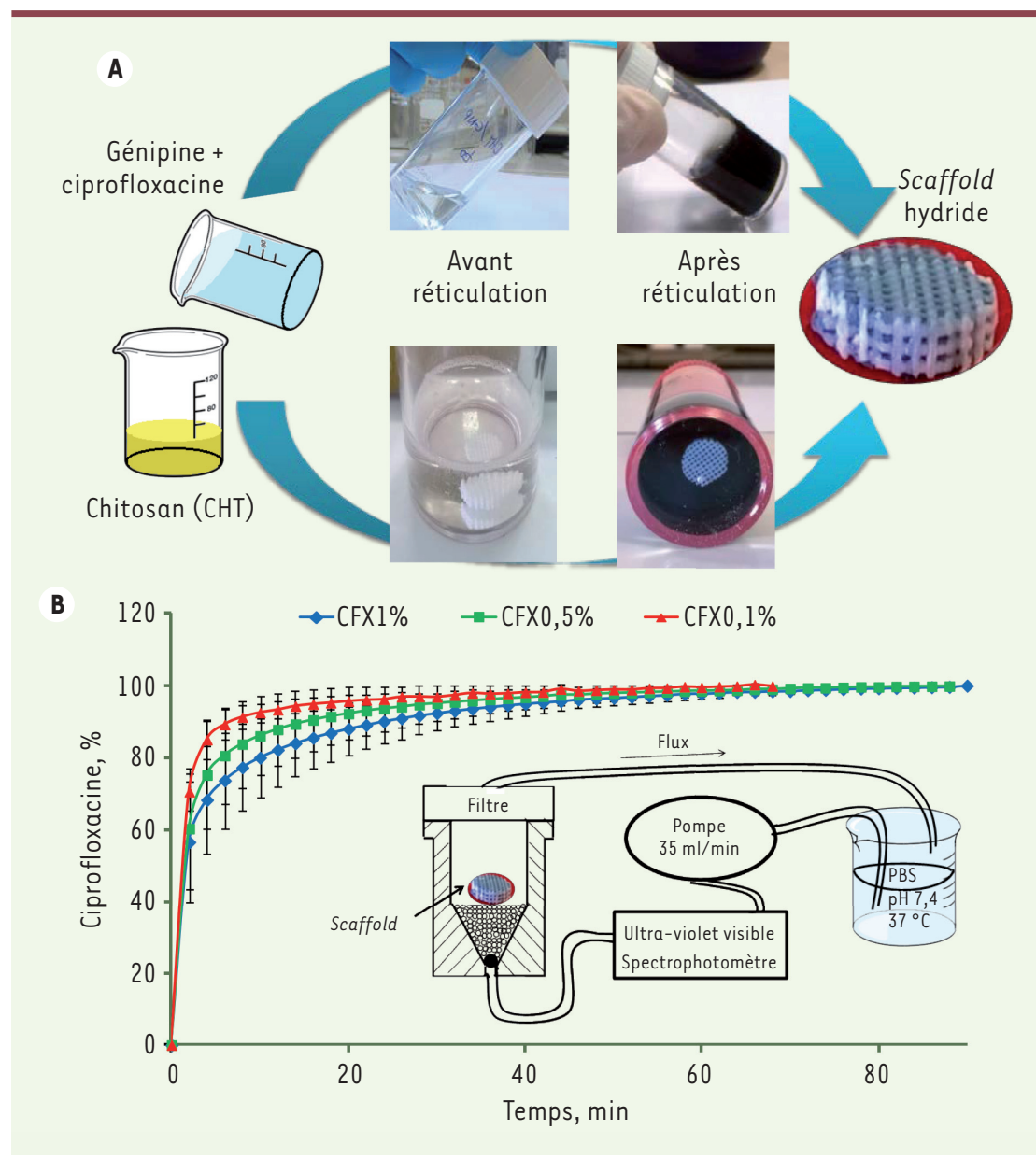

Figure 2. A. Principe de l'incorporation de l'hydrogel de chitosan (CHT) dans la pièce macroporeuse en hydroxyapatite $\left(\varnothing 10 \mathrm{~mm}\right.$, épaisseur $3 \mathrm{~mm}$ ) à $40^{\circ} \mathrm{C}$ pendant 72 heures. La réaction de réticulation du CHT par la génipine provoque une couleur bleutée. B. Profil de libération de la ciprofloxacine (CFX, 0,$1 ; 0,5$ et $1 \%$ ) dans le tampon phosphate (PBS, $\mathrm{pH} 7,4,37^{\circ} \mathrm{C}$ ) en flux continu (35 $\mathrm{ml} / \mathrm{min})$, circuit fermé.

cules thérapeutiques qui ne sont pas sélectives des lignées cellulaires impliquées dans la resténose, ainsi que l'utilisation de polymères non dégradables comme transporteur, pourraient expliquer ces échecs [21].

Depuis une dizaine d'année, la polydopamine (PDA) [22], un polymère bio-inspiré ${ }^{5}$ a été utilisée pour l'immobilisation de nombreuses molécules comme la sélénocystamine ${ }^{6}$ [23], les peptides $\mathrm{RGD}^{7}$ [24] ou le VEGF (vascular endothelial growth factor) [25] sur les surfaces métalliques. Plus récemment la PDA a été revêtue sur des stents en acier inoxydable (316L) par l'équipe de Wang afin d'améliorer la prolifération des cellules endothéliales et ralentir la prolifération des cellules musculaires lisses, cibles de la resténose. Ces travaux ont montré que la PDA pouvait jouer un rôle majeur dans la cicatrisation de la paroi artérielle et dans la réendothélialisation des stents. En collaboration

${ }^{5}$ La PDA est composée de dihydroxyindole, d'indoledione, et de dopamine liées covalemment.

${ }^{6}$ La sélénocystamine possède une structure identique à la cystéine avec un atome de sélénium qui remplace celui de soufre.

${ }^{7}$ Arg-Gly-Asp, ce tripeptide interagit avec les intégrines. avec l'unité Matériaux et Transformation, université de Lille (Dr Joel Lyskawa et $\operatorname{Pr}$ Bernard Martel), un polymère à base de cyclodextrine a été immobilisé sur la couche de PDA afin d'obtenir un stent à libération prolongée de principes actifs. Cette évolution a permis d'obtenir une augmentation significative de la quantité de molécule thérapeutique (le paclitaxel, un agent antimicrotubules) chargée sur le stent et d'offrir un meilleur contrôle de la dose et de la cinétique de libération de ce dernier [26]. Depuis, une amélioration importante a été apportée en créant un système multicouche basé sur l'interaction électrostatique entre le polymère de cyclodextrine chargé négativement et le chitosan, chargé positivement (Figure 3A) [27]. La simvastatine semble être une alternative intéressante au paclitaxel cytotoxique. En effet, en plus de son action première sur le cholestérol, la simvastatine présente des effets pléiotropes comme la diminution de l'agrégation plaquettaire, de l'inflammation et une sélectivité vis-à-vis des cellules endothéliales [28-30]. Les études d'inclusion de la simvastatine dans le système multicouche ont permis de montrer un effet « réservoir ». En effet, la quantité de simvastatine chargée dans le système multicouche passe de 16 à $39 \mu \mathrm{g} / \mathrm{cm}^{2}$ soit un peu plus du double lorsque le nombre de couches passe de 1 à 15 (Figure 3B).

Finalement l'activité thérapeutique des stents a été validée sur un modèle de resténose intra stent développé chez le rat. Le modèle consiste à implanter un stent (d'un diamètre de $2 \mathrm{~mm}$ et d'une longueur de $15 \mathrm{~mm}$ ) dans l'aorte de l'animal pendant 28 jours, temps qui correspond au point critique de la resténose chez le rat. Le ratio néointima/média ${ }^{8}(\mathrm{n} / \mathrm{m})$ a été déterminé sur chacun des stents afin de vérifier leur efficacité. Deux témoins ont été utilisés : un stent nu (témoin positif) et un stent commercial au paclitaxel (témoin négatif). Les résultats ont montré une réduction significative de la resténose sur le stent au paclitaxel versus stent $\mathrm{nu}$ (ratio $\mathrm{n} / \mathrm{m} 0,52$ versus 0,76 ). Le stent chargé de simvastatine a montré des résultats

\footnotetext{
${ }^{8}$ La néointima correspond à l'épaississement de l'intima, couche interne endothéliale du vaisseau sanguin. La média est la couche moyenne du vaisseau, composée de collagène et d'élastine, et contenant des fibres musculaires lisses.
} 


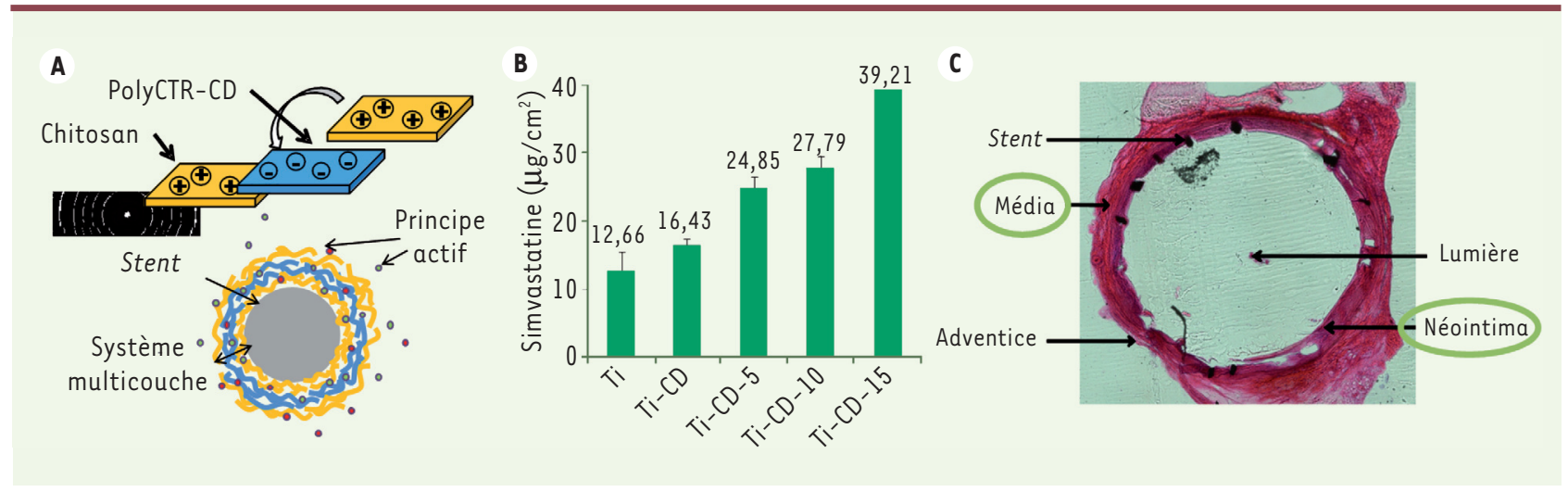

Figure 3. A. Représentation schématique du système multicouche appliqué sur les stents vasculaires. $\boldsymbol{B}$. Quantité de simvastatine adsorbée sur les stents en fonction du nombre de couches. $C$. Représentation d'une coupe obtenue après 28 jours montrant la média et la néointima pour le calcul du ratio $\mathrm{n} / \mathrm{m}$ chez le rat. CTR : acide citrique; $\mathrm{CD}$ : cyclodextrine; Ti : titane.

très encourageants avec une réduction importante du ratio $n / m(0,30)$ comparé au stent nu et au stent paclitaxel. Ces premiers résultats nécessitent néanmoins d'être confirmés par une étude incluant un plus grand nombre d'animaux avant de pouvoir envisager une investigation clinique.

\section{Implants cochléaires}

La délivrance de principes actifs à l'oreille interne est très difficile en raison de la barrière hémato-cochléaire, anatomiquement et fonctionnellement très proche de la barrière hémato-encéphalique [31]. L'administration par des voies classiques (notamment orale, intraveineuse, intramusculaire, intrapéritonéale, intra-articulaire, sous-cutanée, rectale, nasale, etc.), ne permet qu'à de faibles quantités de principe actif d'atteindre le site d'action car le passage de la circulation systémique à l'oreille interne est très fortement entravé par la présence de jonctions serrées (Figure 4A). Des doses élevées en principe actif sont donc requises, potentiellement accompagnées d'effets secondaires graves (dus à des concentrations systémiques considérables en principe actif) et les effets thérapeutiques, quant à eux, restent faibles (ou négligeables), malgré l'existence de principes actifs puissants.

Cette limitation peut efficacement être surmontée par l'administration directe du principe actif au niveau de l'oreille interne. Néanmoins, une telle administration est très délicate car la cochlée est un espace très réduit, clos et sensible à de faibles variations de volume de fluide. De plus, le risque de survenue d'effets secondaires toxiques peut être considérable (en fonction de la dose de principe actif administrée, des cinétiques d'élimination et de la fenêtre thérapeutique). L'élimination du principe actif de l'oreille interne (métabolisme cellulaire local, efflux à travers le point d'injection) et l'absence d'apport continu en principe actif, peut entraîner une chute de sa concentration sous un seuil minimal d'efficacité. L'administration directe à l'oreille interne doit alors être fréquemment répétée ce qui n'est pas envisageable en raison du risque considérable d'infections et de la haute sensibilité du faible volume du liquide de l'oreille interne aux fluctuations. Pour sur- monter ces restrictions, des formes à libération contrôlée peuvent être utilisées [32-35]. L'idée consiste à piéger le principe actif dans une matrice appropriée (souvent des polymères) évitant sa dissolution immédiate. Différents types de phénomènes de transport de masse comme la diffusion de l'eau et du principe actif, ou sa dissolution peuvent être utilisés pour contrôler la vitesse à laquelle il sera libéré du système. Par exemple, un apport constant en principe actif peut être assuré sur de longues périodes compensant ainsi son élimination et permettant le maintien de concentrations constantes au niveau du site d'action. Parallèlement, les concentrations résultantes dans le reste du corps peuvent être minimisées (Figure 4B), ce qui réduit les effets secondaires. Cette approche est particulièrement prometteuse pour de longues périodes de libération (de quelques mois à quelques années).

La Figure $4 C$ montre un exemple pratique : un implant porte-électrode intracochléaire chargé en dexaméthasone préparé par injection moulage (cylindres en silicones contenant des électrodes métalliques de 0,4 et $0,5 \mathrm{~mm}$ de diamètre). Les cinétiques de libération sont illustrées dans la Figure 4D. On peut constater que les vitesses de libération sont très lentes, ce qui est souhaitable dans de nombreuses applications. La performance in vitro et in vivo de ce type d'implants intracochléaires miniaturisés est très prometteuse [33, 34, 37].

\section{Implants parodontaux}

Le traitement de la parodontite ${ }^{9}$ est très complexe car l'affinité du principe actif pour les poches parodontales

${ }^{9}$ Les parodontites sont des lésions du parodonte profond, d'étiologie infectieuse, à
manifestations inflammatoires qui entraînent la destruction des tissus de soutien de la dent. 

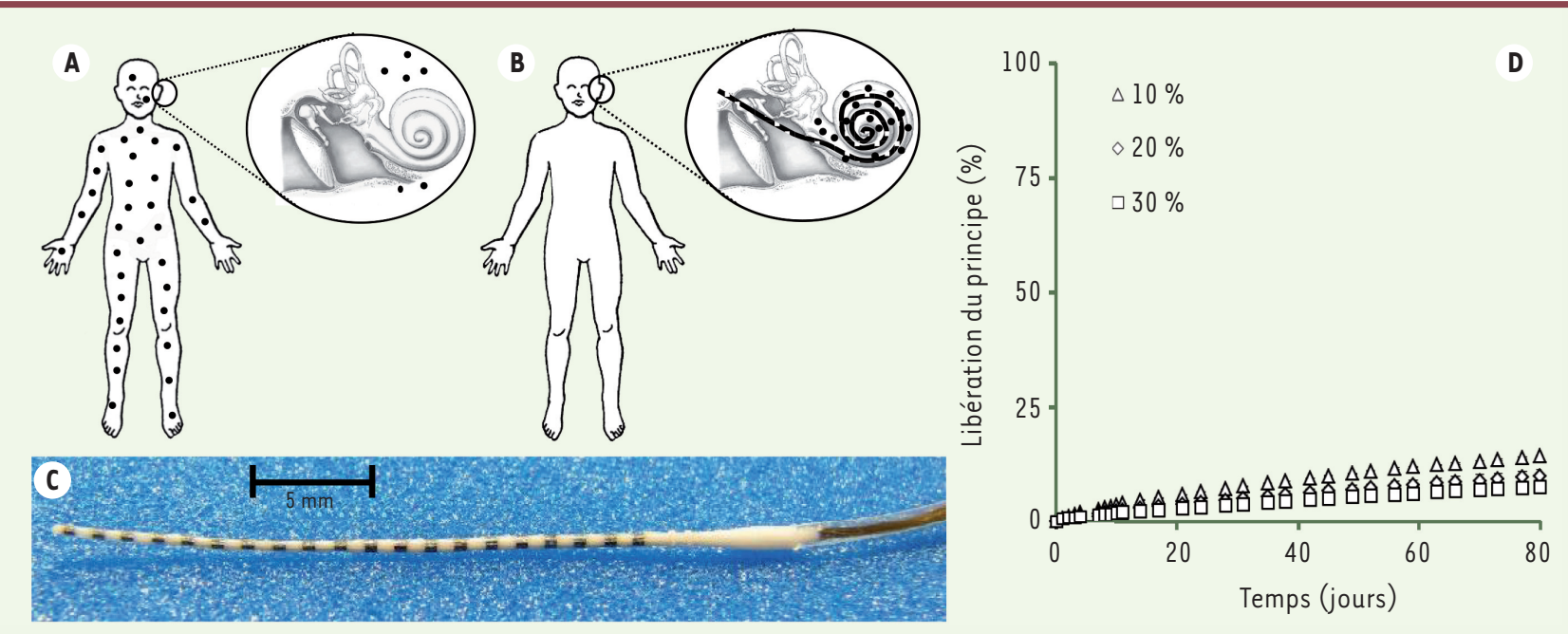

Figure 4. A. Représentation schématique de la distribution en principe actif dans le corps humain après administration par une voie classique (orale, intraveineuse, intramusculaire), ou (B) administration à l'aide d'un implant cochléaire à libération contrôlée. Les points noirs représentent les molécules de principe actif. C. Image macroscopique d'un implant porte-électrode intracochléaire, incorporé dans une matrice de silicone chargée avec $10 \%$ de dexaméthasone. D. Cinétiques de libération à partir de ces implants. La charge en principe actif a été variée de 10 à $30 \%$ (d'après [36] avec la permission de Elsevier).

est généralement très peu prononcée et le flux du fluide gingival créviculaire élimine rapidement les principes actifs du site d'action [38]. II a ainsi été estimé que le contenu d'une poche parodontale de $5 \mathrm{~mm}$ est renouvelé 40 fois par heure [39]. Ainsi le recours à des voies d'administration classiques nécessite souvent de forts taux systémiques de principe actif, ses concentrations au site d'action restant faibles (Figure 5A). Ceci peut conduire à de potentiels effets secondaires graves et une efficacité thérapeutique limitée ou insuffisante malgré l'existence de principes actifs puissants, capables d'agir contre la flore pathogène et l'inflammation.

Ces obstacles d'accessibilité limitée au site d'action et d'élimination rapide peuvent être surmontés grâce à l'utilisation de formes galéniques locales avancées, libérant le principe actif de manière contrôlée dans le temps au sein des poches parodontales [40]. Plusieurs produits visant une telle délivrance locale et contrôlée dans le temps sont disponibles (par exemple: Atridox ${ }^{\circledR}$, Parocline $^{\circledR}$, Elysol $^{\circledast}$ ) mais leurs temps de résidence au niveau du site d'action restent incertains. Il est important de noter que l'utilisation d'antibiotiques pour cette application (systémique ou locale) est très controversée [41]. L’Agence nationale de sécurité du médicament et des produits de santé (ANSM) recommandait fortement, en juillet 2011 , de ne pas utiliser d'antibiotiques dans le traitement de la parodontite chronique, en raison du risque élevé de sélectionner des souches de bactéries résistantes, pour un bénéfice insuffisant [42]. Le recours aux antibiotiques doit donc être limité aux cas où ils sont réellement justifiés. Pour les parodontites, ces cas se limitent aux parodontites agressives ou réfractaires. Pour les parodontites chroniques, les actifs antiseptiques offrent un grand potentiel pour éliminer l'infection anaérobie à la base des poches parodontales et dans les tissus gingivaux inac- cessibles aux instruments parodontaux. Cependant, à ce jour, il existe un réel manque de formes à libération contrôlée pour de tels antiseptiques. Les technologies déjà existantes présentent d'autres désavantages : une élimination prématurée et non-contrôlée de l'implant au cours du traitement, la nécessité d'éliminer les résidus après libération du principe actif, et une géométrie et une taille des implants préformés inappropriées aux besoins des patients. Des implants biodégradables se formant in situ peuvent permettre de surmonter cet obstacle [43]. Il s'agit de formulations liquides qui, après injection dans la poche parodontale, constituent des implants solides (Figure 5B).

Le principe actif et le polymère formant la matrice (par exemple l'acide poly[lactique-co-glycolique], ou PLGA), dissous dans un solvant organique (la N-méthyle pyrrolidone, ou NMP) peuvent facilement être injectés dans les cavités. Le liquide se répandant dans la poche parodontale, un remplissage parfait de la cavité est assuré formant un implant d'une taille et d'une géométrie sur mesure et adaptée à n'importe quelle poche. Une fois injectée, la NMP diffuse dans les tissus environnants et l'eau de la poche parodontale pénètre dans la formulation liquide (Figure 5C). Consécutivement, le PLGA (qui est insoluble dans l'eau) précipite et piège le principe actif. Les implants résultants sont donc adaptés au besoin de chaque patient (en particulier la taille et la géométrie des poches parodontales). Ils libèrent le principe actif de manière contrôlée dans le 


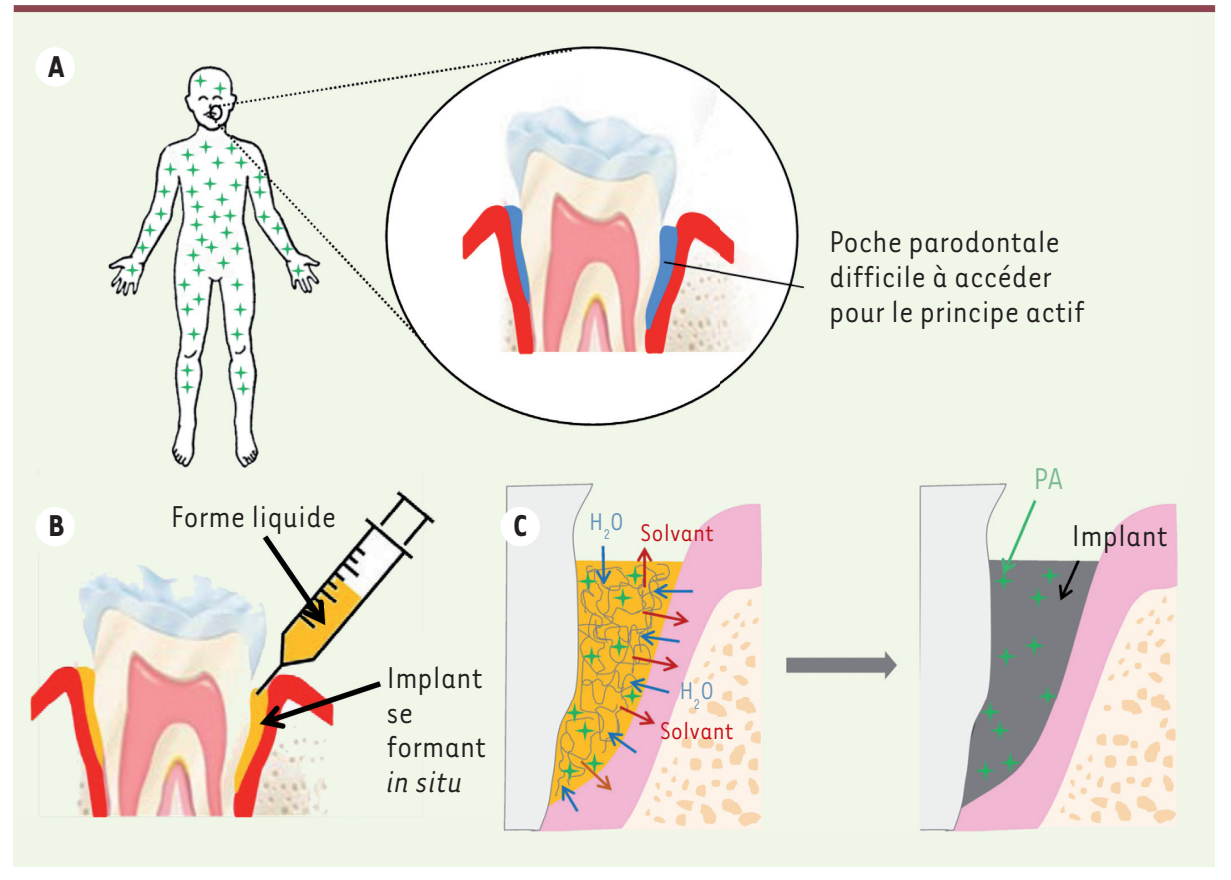

Figure 5. A. Représentation schématique de l'accessibilité limitée aux poches parodontales comme site d'action pour le traitement des parodontites. Les étoiles vertes représentant les molécules de principe actif. B. Représentation schématique de la formation d'un implant se formant in situ dans la poche parodontale après injection d'une formulation liquide à l'aide d'une seringue standard. C. Représentation schématique de la formation d'un implant se formant in situ dans la poche parodontale après diffusion du solvant organique dans les tissus environnants, pénétration de l'eau dans la formulation liquide, précipitation consécutive du polymère et piégeage $d u$ principe actif. Les chaînes représentent les macromolécules de PLGA, les étoiles les molécules de principe actif.

temps, directement au site d'action, et sont biodégradables (ce qui évite la nécessité de retirer les restes de la matrice après libération). Les premiers résultats sur ce type d'implants parodontaux sont très prometteurs [43-45].

\section{Conclusion}

Les implants à libération contrôlée d'un ou de plusieurs principes actifs peuvent offrir d'énormes avantages par rapport aux implants ou formes galéniques classiques. Dans l'avenir on peut attendre des nouveaux produits sur le marché, surmontant les limitations actuellement rencontrées et permettant une délivrance optimisée des principes actifs. $\diamond$

\section{SUMMARY}

\section{Implants for drug substance delivery}

Implants for controlled drug delivery can be very helpful to improve the therapeutic efficacy of a medical treatment, and at the same time reduce the risk of toxic side effects. In this article, four different strategies are exemplarily presented: hybrid bone substitutes combining hydroxyapatite and chitosan hydrogels; vascular stents coated with a bio-inspired polymer; cochlear implants for local dexamethasone delivery; and in-situ forming implants for periodontitis treatment. But this is only a restricted selection, and numerous other approaches and applications based on implants releasing a drug (or a combination of drugs) exist. Compared to conventional implants or pharmaceutical dosage forms, they might offer decisive advantages. $\diamond$

\section{REMERCIEMENTS}

Les auteurs remercient la région Nord Pas de Calais et le FEDER pour le financement des projets au travers des programmes ARCiR «BIOCERamique hybride pour application
MEDicale - Biocermed » et Interreg IVa des 2 mers «MULTIfunctional Drug Eluting Stent - MultiDES» ainsi que l'Agence Nationale de la Recherche pour son support financier ( $N^{\circ}$ ANR-15-CE19-0014-01 et ANR-14-CE16-0025).

\section{LIENS D'INTÉRÊT}

Les auteurs déclarent n'avoir aucun lien d'intérêt concernant les données publiées dans cet article.

\section{RÉFÉRENCES}

1. Préparations parentérales-Implants. Pharmacopée Européenne 8.4, 2014 : 5013-15.

2. Hornez JC, Chai F, Monchau F, et al. Biological and physico-chemical assessment of hydroxyapatite (HA) with different porosity. Biomol Eng $2007 ; 24: 505-9$.

3. Touzet S, Ferri J, Wojcik T, Raoul G. Complications of calvarial bone harvesting for maxillofacial reconstructions. J Craniofac Surg $2011 ; 22$ : 178-81.

4. Romanò $\mathrm{CL}$, Romanò $\mathrm{D}$, Logoluso N, Drago L. Bone and joint infections in adults: a comprehensive classification proposal. Eur Orthop Traumatol 2011; 1: 207-17.

5. Flury BB, Elzi L, Kolbe M, et al. Is switching to an oral antibiotic regimen safe after 2 weeks of intravenous treatment for primary bacterial vertebral osteomyelitis? BMC Infect Dis $2014 ; 14: 226$.

6. Chai F, Hornez JC, Blanchemain N, et al. Antibacterial activation of hydroxyapatite (HA) with controlled porosity by different antibiotics. Biomol Eng 2007 ; 24 : 510-4.

7. Leprêtre S, Chai F, Hornez JC, et al. Prolonged local antibiotics delivery from hydroxyapatite functionalized with cyclodextrin polymers. Biomaterials $2009 ; 30: 6086-93$

8. Schuessele A, Mayr H, Tessmar J, Goepferich A. Enhanced bone morphogenetic protein-2 performance on hydroxyapatite ceramic surfaces. J Biomed Mater Res A 2009; 90 : 959-71.

9. Lee WH, Loo Cy, Rohanizadeh R. A review of chemical surface modification of bioceramics: effects on protein adsorption and cellular response. Colloids Surf B Biointerfaces $2014 ; 122: 823-34$.

10. Xu $Q$, Czernuszka JT, Controlled release of amoxicillin from hydroxyapatitecoated poly(lactic-co-glycoloc acid) microspheres. J Controlled Release 2008 ; $127: 146-53$.

11. Liu H, Li H, Cheng W, et al. Novel injectable calcium phosphate/chitosan composites for bone substitute materials. Acta Biomater $2006 ; 2$ : 557-65. 


\section{RÉFÉRENCES}

12. Berger J, Reist M, Mayer JM, et al. Structure and interactions in covalently and ionically crosslinked chitosan hydrogels for biomedical applications. Eur J Pharm Biopharm 2004 ; 57 19-34.

13. Rinaudo M. Chitin and chitosan: properties and applications. Prog Polym Sci 2006 ; $31: 603-32$

14. Palao-Suaya R, Gómez-Mascaraque LG, Aguilara MR et al. Self-assembling polymer systems fo advanced treatment of cancer and inflammation. Progr Polymer Science $2016 ; 53: 207-48$.

15. Bhattarai N, Gunn J, Zhang M. Chitosan-based hydrogels for controlled, localized drug delivery. Adv Drug Deliv Rev $2010 ; 62: 83-99$.

16. Muzzarelli RAA. Genipin-crosslinked chitosan hydrogels as biomedical and pharmaceutical aids. Carbohydr Polym $2009 ; 77$ : 1-9.

17. Aubert-Viard F, Martin A, Chai, F et al. Chitosan finishing nonwoven textiles loaded with silver and iodide for antibacterial wound dressing applications. Biomed Mater $2015 ; 10: 015023$.

18. Stone GW, Ellis SG, Cox DA, et al. A polymer-based, paclitaxel-eluting stent in patients with coronary artery disease. $N$ Engl J Med $2004 ; 350$ : 221-31.

19. Kastrati A, Mehilli J, Pache J, et al. Analysis of 14 trials comparing sirolimus-eluting stents with bare-metal stents. N Engl J Med 2007 ; 356 : 1030-9.

20. Finn AV, Kolodgie FD, Harnek J, et al. Differential response of delayed healing and persistent inflammation at sites of overlapping sirolimus- or paclitaxel-eluting stents. Circulation 2005 ; $112: 270-8$.

21. Virmani R, Guagliumi G, Farb A, et al. Localized hypersensitivity and late coronary thrombosis secondary to a sirolimus-eluting stent: should we be cautious? Circulation $2004 ; 109$ : 701-5.

22. Lee $H$, Dellatore SM, Miller WM, Messersmith PB. Mussel-inspired surface chemistry for multifunctional coatings. Science $2007 ; 318: 426-30$

23. Weng $Y$, Song 0 , Zhou Y, et al. Immobilization of selenocystamine on Ti02 surfaces for in situ catalytic generation of nitric oxide and potential application in intravascular stents. Biomaterials $2011 ; 32$ : 1253-63.

24. Chien CY, Tsai WB. Poly(dopamine)-assisted immobilization of Arg-Gly-Asp peptides, hydroxyapatite, and bone morphogenic protein-2 on titanium to improve the osteogenesis of bone marrow stem cells. ACS Appl Mater Interfaces $2013 ; 5: 6975-83$.

25. Poh CK, Shi Z, Lim TY, et al. The effect of VEGF functionalization of titanium on endothelial cells in vitro. Biomaterials $2010 ; 31: 1578-85$.

26. Sobocinski J, Laure W, Taha M, et al. Mussel inspired coating of a biocompatible cyclodextrin based polymer onto CoCr vascular stents. ACS Appl Mater Interfaces $2014 ; 6: 3575-86$.

27. Pérez-Anes A, Gargouri M, Laure W, et al. Bioinspired titanium drug eluting platforms based on a Poly- $\beta$-cyclodextrin-chitosan layer-by-layer self-assembly targeting infections. ACS Appl Mater Interfaces $2015 ; 7: 12882-93$.

28. Jaschke B, Michaelis C, Milz S, et al. Local statin therapy differentially interferes with smooth muscle and endothelial cell proliferation and reduces neointima on a drug-eluting stent platform. Cardiovasc Res $2005 ; 68:$ 483-92.

29. Beckman JA, Creager MA. The nonlipid effects of statins on endothelial function. Trends Cardiovasc Med $2006 ; 16: 156-62$.

30. Jacobson JR, Barnard JW, Grigoryev DN, et al. Simvastatin attenuates vascular leak and inflammation in murine inflammatory lung injury. Am J Physiol Lung Cell Mol Physiol 2005 ; 288 : $1026-32$
31. Juhn, S, Rybak L. Labyrinthine barriers and cochlear homeostasis. Acta Otolaryngol $1981 ; 91: 529-34$.

32. Leary Swan $\varepsilon \varepsilon$, Mescher MJ, Sewell WF, et al. Advanced inner ear drug delivery for auditory applications. Adv Drug Deliv Rev 2008 ; 60 : 1583-99.

33. Gehrke M, Sircoglou J, Vincent C, et al. How to adjust dexamethasone mobility in silicone matrices: a quantitative treatment. Eur J Pharm Biopharm 2016; $100: 27-37$.

34. Gehrke M, Sircoglou J, Gnansia D, et al. Ear cubes for local controlled drug delivery to the inner ear. Int J Pharm 2016; $509: 85-94$

35. $\varepsilon$ I Kechai N, Agnely F, Mamelle $\varepsilon$, et al. Recent advances in local drug delivery to the inner ear. Int J Pharm $2015 ; 494: 83-101$.

36. Krenzlin S, Vincent C, Munzke L, et al. Pedictability of drug release from cochlear implants. J Control Rel 2012; 159:60-8.

37. Douchement D, Terranti A, Lamblin J, et al. Dexamethasone eluting electrodes for cochlear implantation: Effect on residual hearing. Cochlear Implants Int $2015 ; 16: 195-200$.

38. Pascale D, Gordon J, Lamster I, et al. Concentration of doxycycline in human gingival fluid. J Clin Periodontol $1986 ; 13: 841-4$

39. Greenstein $\mathrm{G}$, Tonetti M. The role of controlled drug delivery for periodontitis. J Periodontol $2000 ; 71: 125-40$.

40. Schwach-Abdellaoui K, Vivien-Castioni N, Gurny R. Local delivery of antimicrobial agents for the treatment of periodontal diseases. Eur J Pharm Biopharm $2000 ; 50: 83-99$

41. Bonito AJ, Lux L, Lohr KN. Impact of local adjuncts to scaling and root planing in periodontal disease therapy: a systematic review. J Periodontol $2005 ; 76: 1227-36$.

42. ANSM. Recommandations de bonnes pratiques. Prescription des antibiotiques en pratique bucco-dentaire, juillet 2011 (http://ansm.sante. $\mathrm{fr} / v a r / a n s m \_s i t e / s t o r a g e / o r i g i n a l / a p p l i c a t i o n / 753 c 041773 b 2 c e b e a b l e c 25$ bdba06d33.pdf).

43. Do MP, Neut C, Delcourt $\varepsilon$ et al. In situ forming implants for periodontitis treatment with improved adhesive properties. Eur J Pharm Biopharm 2014; $88: 342-50$.

44. Do MP, Neut C, Metz $\mathrm{H}$ et al. Mechanistic analysis of PLGA/HPMC-based in-situ forming implants for periodontitis treatment. Eur J Pharm Biopharm $2015 ; 94: 273-83$

45. Do MP, Neut C, Metz H et al. In-situ forming composite implants for periodontitis treatment: How the formulation determines system performance. Int J Pharm $2015 ; 486: 38-51$.

46. Jordana F, Le Visage C, Weiss P. Substituts osseux. Med Sci (Paris) 2017 ; $33: 60-5$.
TIRÉS À PART

N. Blanchemain

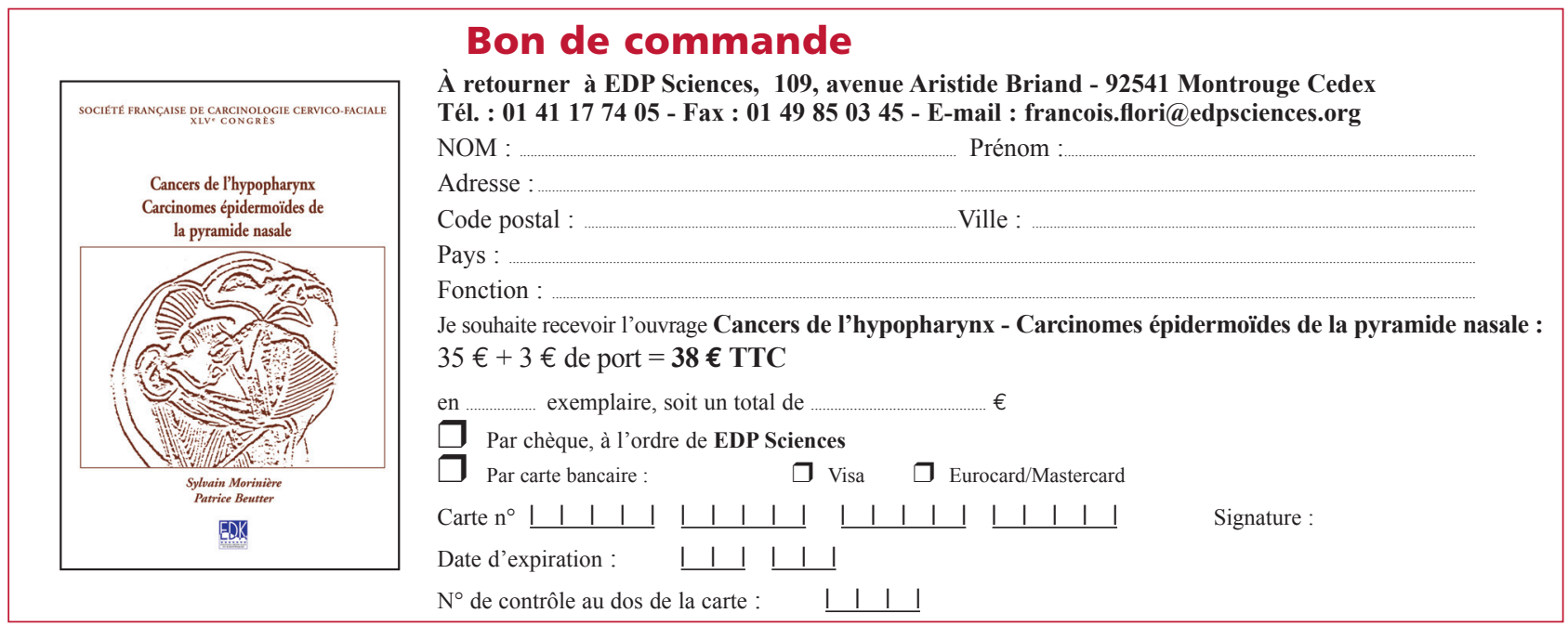

